\title{
Spectral Coincidences Between Emission Lines of the CO Laser and Absorption Lines of Nitrogen Oxides
}

\author{
R. T. MENZIES, MEMBER, IEEE, NICHOLAS GEORGE, SENIOR MEMBER, IEEE, AND MANI L. BHAUMIK, \\ SENIOR MEMBER, IEEE
}

\begin{abstract}
Several spectral coincidences between carbon-monoxide laser emission lines and absorption lines of $\mathrm{NO}$ and $\mathrm{N}_{2} \mathrm{O}_{4}$ have been observed, and absorption measturements have been taken. These data are presented and discussed along with similar spectral coincidence observations made previously using the helium-neon and iodine lasers. Other coincidences between CO-laser lines and $\mathrm{NO}_{2}$ absorption lines are predicted from a study of recently available high-resolution-spectroscopy literature. Due to the narrow line widths of gas-laser lines, the coincidences reported here can be applied to the selective sensing of these constituents in the atmosphere.
\end{abstract}

W E REPORT a study of several spectral coincidences in the $5-6$ - $\mu$-wavelength region between emission lines of the $\mathrm{CO}$ laser and absorption lines of $\mathrm{NO}$ and $\mathrm{N}_{2} \mathrm{O}_{4}$. Due to the fact that gas absorption linewidths are ordinarily very narrow compared with spacings between the lines, cases of overlapping gas-laser lines and molecular gas absorption lines are fortuitous and at the same time valuable in sensing the presence of these resonant constituents in the atmosphere. Cases of spectral coincidence have been reported previously. C. B. Moore has reported this phenomenon with the He-Ne $3.39-\mu\left(3 s_{2} \rightarrow 3 p_{4}\right)$ laser line (absorption by methane) [1]. $P$. Hanst has indicated similar coincidences with the $\mathrm{CO}_{2}$-laser lines at $9.5 \mu$ (absorption by ozone) [2], and the iodine-laser lines at $4.862 \mu$ (absorption by carbon monoxide) and $5.497 \mu$ (absorption by NO) [2].

The liquid-nitrogen-cooled $\mathrm{CO}$ laser emits a multitude of lines at high-power levels in the $5-7-\mu$ region [3], [4], and the room-temperature $\mathrm{CO}$ laser also emits several lines, most of them being at the longer wavelengths [5], [6]. Using published data on the nitric oxide fundamental absorption band [7] and the CO-laser emissionline data of C. K. N. Patel [3], and unpublished data of W. B. Lacina [6], we predicted several spectral coincidences. Some of these coincidences were observed using the $\mathrm{CO}$ laser, both at liquid-nitrogen temperature and at room temperature. In addition, certain room-temperature CO-laser lines in the 5.6-5.7- $\mu$ region were found to be strongly absorbed by $\mathrm{N}_{2} \mathrm{O}_{4}$.

The absorption data were taken by placing a $10-\mathrm{cm}$ absorption cell with $\mathrm{NaCl}$ windows in the path of the

Manuscript received June 1970; revised August 18, 1970. This work was supported in part by the Air Force Office of Scientific Research and the California Institute of Technology, Pasadena, Calif.

R. T. Menzies and N. George are with the California Institute of Technology, Pasadena, Calif. 91109.

M. L. Bhaumik is with the Northrop Corporate Laboratories, Hawthorne, Calif, 90250 . laser beam and passing the beam through a Jarrell-Ash one-meter Czerny-Turner spectrometer with a liquidnitrogen-cooled InSb detector at the exit slit. Data were taken both for pure gases and for the gases in atmosphericpressure backgrounds, in order to observe the effects of broadening and shifts of the absorption lines due to high pressures of foreign gases. The pressure-broadened linewidths at one atmosphere are quite large (about 0.1-0.2 $\mathrm{cm}^{-1}$ [8], [9]). Since NO is oxidized by air, studies of foreign-gas broadening of the NO lines were made with high-purity nitrogen as a background gas. The $\mathrm{N}_{2} \mathrm{O}_{4}$ absorption was then studied using air as a background gas.

In gaseous form, $\mathrm{NO}_{2}$ and $\mathrm{N}_{2} \mathrm{O}_{4}$ exist together, their relative proportions depending on the total pressure and the temperature. At typical atmospheric concentrations, the predominant component is $\mathrm{NO}_{2}$ and therefore absorption data for $\mathrm{N}_{2} \mathrm{O}_{4}$ are probably not particularly relevant in the detection of atmospheric pollutants. However, they may be important in the study of reaction rates and high-resolution spectroscopy. At a total pressure of 50 torr, the ratio of $\mathrm{NO}_{2}: \mathrm{N}_{2} \mathrm{O}_{4}$ is about $7: 3$ at room temperature, and it increases with increasing temperature. The observed $\mathrm{N}_{2} \mathrm{O}_{4}$ absorption of the CO-laser lines disappears as the absorption cell is heated to $140^{\circ} \mathrm{C}$, verifying that this absorption is due entirely to $\mathrm{N}_{2} \mathrm{O}_{4}$ and not $\mathrm{NO}_{2}$.

Measurements of the absorption of the $3.3923-\mu \mathrm{He}-\mathrm{Ne}$ laser line have been reported previously in a different context [1], [10]. The pressure broadening of this particular methane absorption line $\left[\nu_{3}\right.$ band, $\left.\mathrm{P}(7)\right]$ by various foreign gases has also been studied, using Zeeman-tuned multimode lasers [11], [12]. We have studied this methane absorption line using a short $\mathrm{He}-\mathrm{Ne}$ laser that could be operated in single $\mathrm{TEM}_{00 \mathrm{a}}$ mode fashion at $3.3923 \mu$ while being tuned over a region of $\pm 150 \mathrm{MHz}$ from its line center [13].

Table I lists the data obtained using the CO laser, along with corresponding spectral coincidence data using the helium-neon and iodine lasers. All three of the absorbing gases listed play an important part in atmospheric photochemistry and the production of photochemical smog [14]. Several absorption measurements were made using pressures of pure NO in the 10-100-torr range. Results at two pressures, 20 and 50 torr, are shown. Since the Doppler width of the NO absorption line at room temperature is about $125 \mathrm{MHz}$ (full width at half-maximum intensity), and the pressure broadening is of the order of $10 \mathrm{MHz}$ / torr, the absorption lines for these pressures are partially 
TABLE I

Spectral Coincidences Between Laser Lines and Moleculat Absorbers

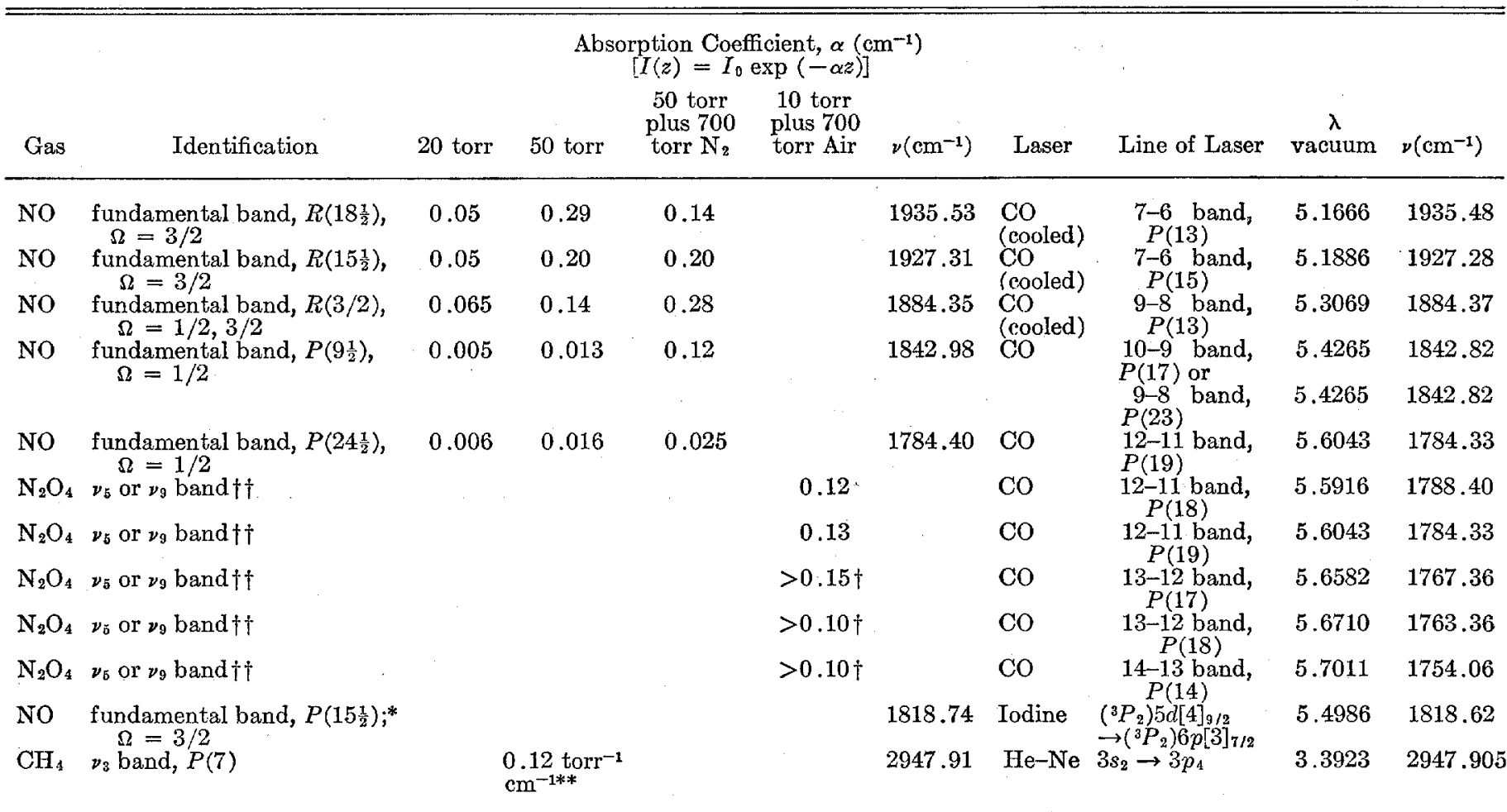

* Probable identification of P. Hanst's observation [2].

** At center of Doppler-broadened absorption line.

$\dagger$ The uncertainty in these data is caused by a weak relatively unstable laser output at this wavelength.

$\dagger \dagger$ The rotational line spacing in this vibration-rotation band [ $\left.\lesssim 0.5 \mathrm{~cm}^{-1}\right]$ is too small for us to make precise identifications of the absorption lines.

pressure broadened. (The pressure-broadening coefficient for the methane line previously mentioned has been reported to be $16 \mathrm{MHz} /$ torr [15].)

For low pressures of an absorbing gas (not greater than one atmosphere), the lineshape near line center should take the form

$$
\alpha_{\nu}=\alpha_{0} \frac{a}{\pi} \int_{-\infty}^{\infty} \frac{\exp \left(-y^{2}\right) d y}{a^{2}+(\omega-y)^{2}}
$$

or

$$
\alpha_{\nu} / \alpha_{0}=\operatorname{Re} w^{*}(\omega+i a)
$$

where $y$ and $\omega$ are the frequency displacements from line center $\nu_{0}$, and $a$ is the sum of the natural and pressurebroadening linewidths, all normalized by the Doppler linewidth [16]; and $w(\omega+i a)$ is the error function for complex arguments [17]. The quantity $\alpha_{0}$ depends on the line strength and the number of absorbing molecules [16]. If one measures the absorption at line center for this case, at pressures less than a torr, one can obtain an absorption coefficient that is linearly proportional to pressure.

Our data for $\mathrm{NO}$ absorption are not at the centers of the lines, since the spectral coincidences are not exact, and we had no means of tuning the CO-laser lines in order to find the line centers. In addition, at pressures ranging from 10 to 100 torr, where we took our data, shifts and broadenings of absorption lines due to collision effects can appear. Consequently we present our data at representative pressures and refrain from drawing any quantitative conclusions concerning the values of the absorption coefficients at the centers of the lines. On the other hand, the methane absorption measurements were taken at much lower pressures, and we had the ability to tune the laser line to the peak of the absorption line, which entailed a frequency shift of about $125 \mathrm{MHz}$. Thus the absorption coefficient for the methane line presented in Table I is the result of data taken at pressures below 1 torr and at the center of the predominantly Doppler-broadened absorption line.

The $\nu_{3}$ absorption band of $\mathrm{NO}_{2}$ ranges from 1550 to $1650 \mathrm{~cm}^{-1}$, which falls in the wavelength region covered by the $Q$-switched liquid-nitrogen cooled $\mathrm{CO}$ laser. The recent accurate measurements of the emission lines of this laser by Mantz et al. [4] have made possible the prediction of several coincidences between these laser lines and the absorption lines of $\mathrm{NO}_{2}$ [18]. Coincidences between $\mathrm{CO}$ laser lines and $\mathrm{NO}_{2}$ absorption peaks that are within $\pm 0.01 \mathrm{~cm}^{-1}$ are listed in Table II. A few of these coincidences might be close enough so that the laser lines could be tuned to exact coincidence with the $\mathrm{NO}_{2}$ absorption peaks using a piezoelectric translator (PZT) on one mirror.

With atmospheric-pressure broadening, a coincidence within about $\pm 0.1 \mathrm{~cm}^{-1}$ would ensure absorption of the 
TABLE II

Coincidences Between Measured CO Laser Lines and ABsorption Lines OF THE $\nu_{3}$ Band OF $\mathrm{NO}_{2}$ то W IтHIN $\pm 0.01 \mathrm{CM}^{-1}$

\begin{tabular}{|c|c|}
\hline Line of Laser & $\nu\left(\mathrm{cm}^{-1}\right)$ \\
\hline $\begin{array}{ll}18-17 \text { band, } & P(13) \\
19-18 \text { band, } & P(8) \\
20-19 \text { band, } & P(6) \\
20-19 \text { band, } & P(10) \\
22-21 \text { band, } & P(10) \\
23-22 \text { band, } & P(8) \\
23-22 \text { band, } & P(11)\end{array}$ & $\begin{array}{l}1658.23 \\
1651.30 \\
1633.31 \\
1619.57 \\
1570.37 \\
1552.62 \\
1542.46\end{array}$ \\
\hline
\end{tabular}

CO-laser line by $\mathrm{NO}_{2}$. Since the spacing of the $\mathrm{NO}_{2}$ absorption lines throughout most of this band is $0.2 \mathrm{~cm}^{-1}$ or less, almost every laser line in this wavelength region would be absorbed to some extent by $\mathrm{NO}_{2}$ in an atmospheric background. The detection of $\mathrm{NO}_{2}$ in the atmosphere is further complicated by strong water-vapor absorption in this spectral region [18].

It would be profitable to conduct more extensive studies of these absorption lines with the use of an external frequency shifter. Since the Doppler-gain profiles of the CO-laser lines have widths of about $100-200 \mathrm{MHz}$, depending on the mode of operation, the tuning range of a PZT mirror scan is limited. The CO laser used in these measurements did not have a PZT mirror scanner, thus the accuracy of our absorption data for the 20-torr column of Table $I$ is limited due to frequency drifts of the laser lines about their respective line centers. At higher gas pressures, the absorption linewidths are much larger than the Doppler widths of the laser-line gain profiles, making the absorption data less sensitive to drifts caused by slight changes in the laser-cavity length.

\section{ACKNOWLEDGMENT}

The authors wish to thank J. Walder for his helpful discussions and M. S. Shumate and G. S. Levy for their encouragement. The experimental results with the
CO laser were obtained at the Northrop Corporate Laboratories. They also appreciate the technical assistance of D. Diem and D. Pace and thank Prof. K. Narahari Rao for permitting the use of [4] and [18] prior to their publication.

\section{REFERENCES}

[1] C. B. Moore, "Gas laser frequency selection by molecular absorption," Appl. Opt., vol. 4, pp. 252-253, February 1965.

[2] P. L. Hanst, "Infrared spectroscopy and infrared lasers in air pollution research and monitoring," Appl. Spectrosc., vol. 24, pp. 161-174, March 1970.

[3] C. K. N. Patel, "Vibrational-rotational laser action in carbon monoxide," Phys. Re"., vol. 141, pp. 71-83, January 1966.

[4] A. W. Mantz, E. R. Nichols, B. D. Alpert, and K. N. Rao, "CO laser spectra with a 10-meter vacuum infrared grating spectrograph," J. Mol. Spectrosc., vol. 35, pp. 325-328, August 1970 .

[5] M. M. Mann, M. L. Bhaumik, and W. B. Lacina, "Roomtemperature CO laser," Appl. Phys. Lett., vol. 16, pp. 430431 , June 1970 .

[6] W. B. Lacina, M. M. Mann, and M. L. Bhaumik (to be published).

[7] J. H. Shaw, "Nitric oxide fundamental," J. Chem. Phys., vol. 24, pp. 399-402, February 1956.

[8] D. H. Rank, U. Fink, J. V. Foltz, and T. A. Wiggins, "Intensity measurements on spectra of gases of planetary interest$\mathrm{H}_{2}, \mathrm{H}_{2} \mathrm{O}$, and $\mathrm{CO}_{2}, "$ Astrophys. $J$., vol. 140, pp. 366-373, 1964 .

[9] W. S. Benedict and L. A. Kaplan, "Calculation of line widths in $\mathrm{H}_{2} \mathrm{O}-\mathrm{N}_{2}$ collisions," $J$. Chem. Phys., vol. 30, pp. 388-399, 1959.

[10] R. T. Menzies, A. Dienes, and N. George, "Axial magnetic field effects on a saturated He-Ne laser amplifier," IEEE $J$. Quantum Electron., vol. QE-6, pp. 117-122, February 1970.

[11] H. J. Gerritsen and M. E. Heller, "High-resolution tunedlaser spectroscope," Appl. Opt. Suppl. pt. 2, p. 73, 1965.

[12] H. Goldring, A. Szöke, E. Zamir, and A. Ben-Reuven, "Pressure broadening and shift of $3.39-\mu$ absorption in methane perturbed by noble gases," J. Chem. Phys., vol. 49, p. 4253, 1968.

[13] R. Menzies, "Magnetic field and pressure effects in a saturated gas laser amplifier," Ph.D. dissertation, California Institute of Technology, Pasadena, Sci. Rept. 11, AFOSR-68-1492, 1970 .

[14] R. D. Cadle and E. R. Allen, "Atmospheric photochemistry," Science, vol. 167, pp. 243-249, January 1970.

[15] R. L. Barger and J. L. Hall, "Pressure shift and broadening of methane line at $3.39-\mu$ studied by laser-saturated molecular absorption," Phys. Rev. Letl., vol. 22, pp. 4-7, 1969.

[16] A. C. G. Mitchell and M. W. Zemansky, Resonance Radiation and Excited Atoms. New York: Cambridge University Press, 1961.

[17] M. Abramowitz and I. A. Stegun, Eds., Handbook of Mathematical Functions, Appl. Math. Ser. 55. Washington, D. C.: NBS, 1964, pp. 297-304, 325-328.

[18] S. C. Hurlock, Ph.D. dissertation, Ohio State University, Columbus, 1970 . 